\title{
Musgos de bosques húmedos de montaña en la Sierra Madre Oriental: Nuevos ReGistros ReGionales
}

\author{
Patricia Herrera-Paniagua ${ }^{1}$ y Mahinda Martínez \\ Escuela de Biología, Universidad Autónoma de Querétaro, Juriquilla, Querétaro, México \\ ${ }^{1}$ Autor para la correspondencia: hepatyca@hotmail.com
}

\begin{abstract}
Resumen: Se registran 23 especies de musgos nuevos para la flora de los estados de Querétaro y San Luis Potosí, de distribución tanto restringida como amplia. Destacan Cyclodictyon richardsii y Pylaisiadelpha sharpii por ser plantas inconspicuas y endémicas al país, además de Donnellia commutata, conocida para México, pero sin material mexicano en los herbarios nacionales. Se discuten las características taxonómicas distintivas de las especies, su hábitat y distribución.

Palabras clave: biodiversidad, epífitas, Querétaro, San Luis Potosí, Sierra Gorda.
\end{abstract}

\begin{abstract}
Twenty-three new records for moss species are reported from the states of Querétaro and San Luis Potosí, some with wide geographic ranges but others with restricted ranges. Among the restricted species, Cyclodictyon richardsii and Pylaisiadelpha sharpii are inconspicuous and endemic to Mexico. Donnellia commutata is known from Mexico but vouchers are lacking in national collections. Taxonomic characteristics, distribution and habitat of the new records are discussed.
\end{abstract}

Key words: biodiversity, epiphytes, Querétaro, San Luis Potosí, Sierra Gorda.

$\mathbf{P}$ ara los estudios de musgos en México, la obra de Sharp et al. (1994) es una base importante por los datos taxonómicos y de distribución de las especies que contiene. Sin embargo, y a pesar de las áreas estudiadas en diferentes partes del país (véanse, por ejemplo, Delgadillo 1984; Delgadillo y Cárdenas, 1996; Cárdenas, 1999), aún hay lugares y hábitats cuyo conocimiento brioflorístico es parcial o donde no hay ningún estudio formal; por ejemplo, los estados de Aguascalientes y Tabasco (Delgadillo, 1998). El estado de Querétaro también se encontraba en esta situación, hasta que Herrera-Paniagua et al. (2008) hicieron un estudio en el que se mostró que la flora de musgos comprendía 212 especies. El estudio incluyó la recolecta en todos los municipios y tipos de vegetación del estado, pero se consideró que el número podría incrementarse con la exploración minuciosa de lugares tan diversos como la Sierra Gorda. Otros estados como San Luis Potosí, han recibido más atención por parte de los investigadores, lo que incluye la descripción de algunas especies nuevas para la ciencia (Ando y Higuchi, 1983). La flora de musgos de México (Sharp et al., 1994) cita 210 especies para San Luis Potosí, pero hay varias zonas sin explorar (p. ej. el suroeste), por lo que su conocimiento florístico también se considera parcial.
En México son pocos los estudios de musgos en bosques húmedos de montaña (BHM, sensu Villaseñor, 2010), como los de Delgadillo (1979) y Juárez (1983), quienes citan 194 y 146 especies respectivamente. Sobre epífitas existe sólo un trabajo que incluye una lista de 54 especies presentes en ramas de árboles de algunos BHM y selvas (Thornburgh y Sharp, 1975). Por lo anterior, se ha desarrollado un estudio en torno a los musgos epífitos en BHM de la región terrestre prioritaria Sierra Gorda-Río Moctezuma (RTP 101; Arriaga et al., 2000) de los estados de Querétaro y San Luis Potosí. Como parte del estudio se han identificado nuevos registros para la región. Algunos de ellos son especies mal representadas en el Herbario Nacional (MEXU) y parecen tener una distribución restringida. Para otras se amplía su área de distribución. La identificación taxonómica fidedigna y la ubicación de estas especies permiten establecer con mayor precisión sus áreas de distribución, lo cual es relevante para situar zonas prioritarias a conservar (Hallingbäck y Hodgetts, 2000). En este trabajo se proporciona una breve descripción de los nuevos registros, se señalan sus características más distintivas, microhábitat, afinidad florística y su distribución en el país. 


\section{Materiales y métodos}

De octubre del 2009 a octubre del 2011 se colectaron las epífitas en cuatro fragmentos de BHM (Figura 1), en las localidades de Copalillos (21 51' 12.18' N, 99 27' 9.18' O, 1,252 m s.n.m.) y El Retén ( $21^{\circ} 17^{\prime} 49.38^{\prime \prime}$ N, $99^{\circ} 85^{\prime} 10.98^{\prime \prime}$ O, 1,485 m s.n.m.), en San Luis Potosí. En Querétaro se colectaron ejemplares en Los Xililites ( $21^{\circ} 28^{\prime} 27.84^{\prime \prime} \mathrm{N}, 99^{\circ} 09^{\prime}$ 52.32" O, 1,294 m s.n.m.) y Pozo del Limón (21 15' 26.8” $\mathrm{N}, 9^{\circ} 03$ ' 17.5" O, $989 \mathrm{~m}$ s.n.m.). Las muestras provienen de los troncos de árboles (0-8 $\mathrm{m}$ de altura), excepto un musgo que se colectó sobre suelo. Se incluyen tres ejemplares colectados por A. Cárdenas en Ahuacatlán ( $21^{\circ} 18^{\prime} \mathrm{N}, 99^{\circ} 03^{\prime}$ O, San Luis Potosí) y en La Cercada $\left(21^{\circ} 28^{\prime} \mathrm{N}, 9^{\circ} 07^{\prime} \mathrm{O}\right.$, Querétaro) por S. Zamudio y V. Steinman (Figura 1).

Los ejemplares se determinaron en el laboratorio, según Sharp et al. (1994), Buck (1998), Allen y Magill (2007) y Pursell (2007). La nomenclatura y los datos de distribución mundial están conforme a la versión electrónica de LATMOSS (Delgadillo, 2010) e información de distintos herbarios proporcionada por GBIF (2013). Los datos de la distribución nacional se tomaron de Sharp et al. (1994) y de la UNIBIO (2012). Los elementos fitogeográficos siguen el criterio de Delgadillo $(1987,2004)$ con la modificación del concepto Mesoamericano que se consideró para las especies que se presentan sólo en México y Centro América. Los ejemplares están depositados en el Herbario de la Uni-

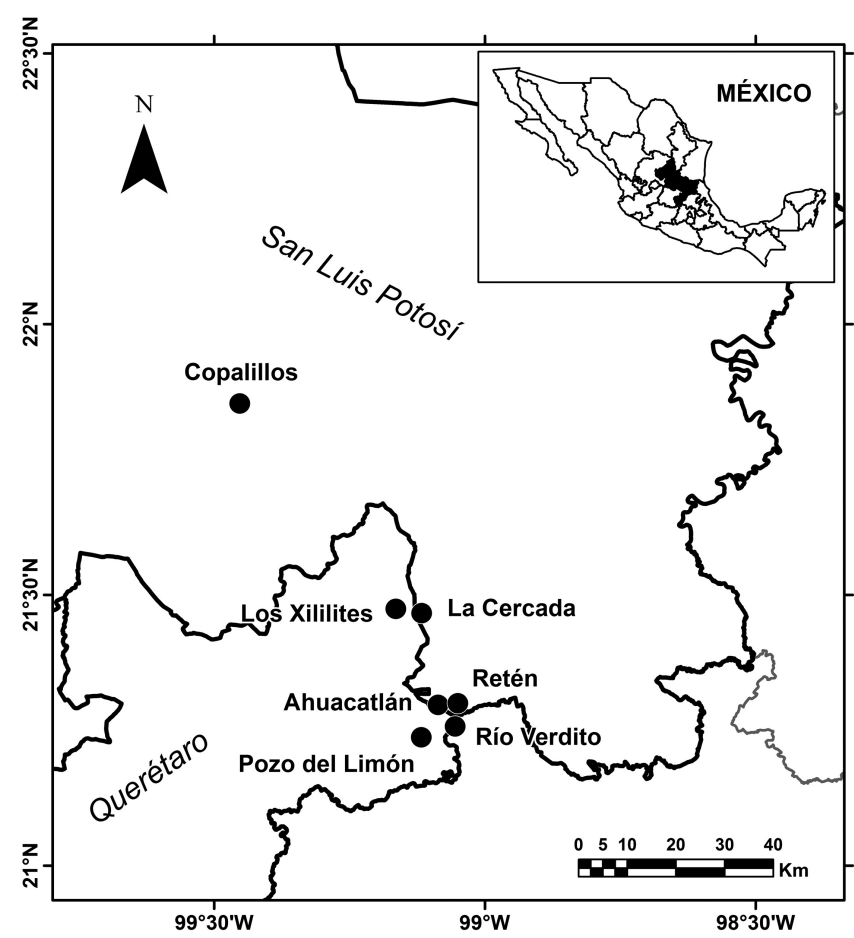

Figura. 1. Localización de los fragmentos de bosque húmedo de montaña (BHM) estudiados en los estados de San Luis Potosí y Querétaro, México. versidad Autónoma de Querétaro "Dr. Jerzy Rzedowski" (QMEX), excepto los ejemplares de Cárdenas y de Zamudio y Steinmann que se encuentran en la Colección de Briofitas del Herbario Nacional (MEXU).

\section{Resultados}

En total se contabilizaron 23 nuevos registros, de los cuales 12 son nuevas especies para la flora de musgos del estado de Querétaro, cinco para San Luis Potosí y seis para ambos. Por su distribución mundial (Cuadro 1), sólo ocho de estas especies mostraron estar bien representadas en el mundo: Isopterygium tenerum (Sw.) Mitt. se presenta en varios continentes (amplia distribución); Hildebrandtiella guyanensis (Mont.) W.R.Buck y Syrrhopodon incompletus Schwägr. var. incompletus se encuentran tanto en América como en África (amplia distribución bicéntrica); Fissidens serratus Müll.Hal. var. serratus, Syrrhopodon parasiticus (Brid.) Besch. y Syrrhopodon prolifer Schwägr. var. prolifer se encuentran en las principales zonas tropicales del mundo (pantropicales); y Anomodon rostratus (Hedw.) Schimp. y Fissidens dubius P.Beauv. se registran en el Hemisferio Norte (boreales). El resto de las especies (15) sólo se conocen en el continente Americano, presentan registros en varios países, sin incluir a las Antillas (Americanas; cuatro) o incluyéndolas (del Caribe; ocho). Sólo Sphaerotheciella pachycarpa (Schimp. ex Besch.) Manuel se encuentra tanto en México como en Centroamérica (estrictamente Mesoamericana) y Cyclodictyon richardsii Bowers \& Magill y Pylaisiadelpha sharpii H.A.Crum están restringidas al país (endémicas).

Anomodon rostratus (Hedw.) Schimp., Syn. Musc. Eur. 488. 1860. (Anomodontaceae). Se caracteriza por su hojas ovadas, acuminadas y aristadas, con márgenes revolutos y células densamente papilosas (Buck, 1998). Algunos ejemplares colectados mostraron variaciones morfológicas, como carecer de las hojas aristadas, con los márgenes ligeramente revolutos, aurículas ligeramente desarrolladas y células foliares arregladas en series regulares y las células basales sin papilas. Tales variaciones pueden ser producto del estado juvenil de las plantas (C. Delgadillo, com. pers.).

Distribución y hábitat. Troncos de Liquidambar styraciflua L. Es una especie de distribución boreal (Delgadillo, 2004) y en México se le ha encontrado desde Nuevo León y Tamaulipas (Rancho El Cielo) hasta Chiapas (UNIBIO, 2012). Ejemplares examinados. Querétaro. Mpio. Landa de Matamoros, Pozo del Limón, aprox. $3 \mathrm{~km}$ al sur de Neblinas, 22 de agosto de 2009, P. Herrera $1187 b$.

Atrichum oerstedianum (Müll.Hal.) Mitt., J. Linn. Soc., Bot. 12: 605.1869. (Polytrichaceae). Son plantas robustas que se distinguen por sus hojas rizadas cuando secas, erectas y esparcidas cuando húmedas, no envainantes en la base, la costa con lamelas de 1-3 células de alto y células lisas (Cárdenas y Delgadillo, 2009). 
Cuadro 1. Distribución mundial generalizada de los nuevos registros de musgos epífitos de bosques húmedos de montaña para los estados de Querétaro y San Luis potosí. Las especies pueden estar en todos, en varios o en un solo país de las zonas señaladas, para más detalles refiérase a LATMOSS (Delgadillo, 2010) y al portal GBIF (2013). NA1 (Canadá), NA2 (Estados Unidos), NA3 (México), CA (Centro América), SA1 (Colombia, Venezuela, Guyana, Surinam, Guyana Francesa), SA2 (Ecuador, Perú, Bolivia, Brasil, Islas Galápagos), SA3 (Chile, Paraguay, Argentina, Uruguay, Islas Juan Fernández), ANT (Antillas Mayores y Menores), AFR (África), EUR (Europa), AS (Asia), OC (Oceanía).

\begin{tabular}{|c|c|c|c|c|c|c|c|c|c|c|c|c|}
\hline Taxa & NA1 & NA2 & NA3 & CA & SA1 & SA2 & SA3 & ANT & AFR & EUR & AS & OC \\
\hline $\begin{array}{l}\text { Anomodontaceae } \\
\text { Anomodon rostratus (Hedw.) Schimp. }\end{array}$ & $x$ & $X$ & $X$ & $x$ & & & & $X$ & & $x$ & $x$ & \\
\hline $\begin{array}{l}\text { Calymperaceae } \\
\text { Syrrhopodon incompletus Schwägr. var. incompletus } \\
\text { Syrrhopodon parasiticus (Brid.) Besch. } \\
\text { Syrrhopodon prolifer Schwägr. var. prolifer }\end{array}$ & & $\begin{array}{l}X \\
X\end{array}$ & $\begin{array}{l}X \\
X \\
X\end{array}$ & $\begin{array}{l}X \\
X \\
X\end{array}$ & $\begin{array}{l}X \\
X \\
X\end{array}$ & $\begin{array}{l}X \\
X \\
X\end{array}$ & $\begin{array}{l}X \\
X \\
X\end{array}$ & $\begin{array}{l}X \\
X \\
X\end{array}$ & $\begin{array}{l}X \\
X \\
X\end{array}$ & & $\begin{array}{l}x \\
x\end{array}$ & $\begin{array}{l}x \\
x\end{array}$ \\
\hline $\begin{array}{l}\text { Cryphaceae } \\
\text { Sphaerotheciella pachycarpa (Schimp. ex Besch.) Manuel }\end{array}$ & & & $x$ & $X$ & & & & & & & & \\
\hline $\begin{array}{l}\text { Dicranaceae } \\
\text { Campylopus anderssonii (Müll.Hal.) Jaeg. }\end{array}$ & & & $x$ & $x$ & $X$ & $X$ & & & & & & \\
\hline $\begin{array}{l}\text { Entodontaceae } \\
\text { Entodon hampeanus Müll.Hal. } \\
\text { Entodon jamesonii (Taylor) Mitt. } \\
\text { Entodon serrulatus Mitt. }\end{array}$ & & $x$ & $\begin{array}{l}X \\
X \\
X\end{array}$ & $\begin{array}{l}X \\
X \\
X\end{array}$ & $\begin{array}{l}X \\
X\end{array}$ & $\begin{array}{l}X \\
X\end{array}$ & & $\begin{array}{l}X \\
X\end{array}$ & & & & \\
\hline $\begin{array}{l}\text { Fissidentaceae } \\
\text { Fissidens dubius P.Beauv. } \\
\text { Fissidens serratus Müll.Hal. var. serratus }\end{array}$ & $x$ & $\begin{array}{l}X \\
X\end{array}$ & $\begin{array}{l}X \\
X\end{array}$ & $\begin{array}{l}X \\
X\end{array}$ & $X$ & $X$ & $x$ & $\begin{array}{l}X \\
X\end{array}$ & $X$ & $x$ & $\begin{array}{l}X \\
X\end{array}$ & $x$ \\
\hline $\begin{array}{l}\text { Hypnceae } \\
\text { Isopterygium tenerum (Sw.) Mitt. }\end{array}$ & & $X$ & $x$ & $x$ & $X$ & $x$ & $x$ & $x$ & $X$ & $x$ & $x$ & \\
\hline $\begin{array}{l}\text { Leucobryaceae } \\
\text { Leucobryum crispum Müll.Hal. }\end{array}$ & & & $X$ & $x$ & $X$ & $X$ & $x$ & $X$ & & & & \\
\hline $\begin{array}{l}\text { Meteoriaceae } \\
\text { Squamidium leucotrichum (Taylor) Broth. }\end{array}$ & & & $x$ & $x$ & $X$ & $X$ & & $X$ & & & & \\
\hline $\begin{array}{l}\text { Neckeraceae } \\
\text { Orthostichella pachygastrella (Müll.Hal. ex Ångstr.) } \\
\text { B.H.Allen \& Magill }\end{array}$ & & & $x$ & $x$ & & $x$ & $x$ & & & & & \\
\hline $\begin{array}{l}\text { Orthotrichaceae } \\
\text { Schlotheimia jamesonii (Arnott) Brid. }\end{array}$ & & & $x$ & $x$ & $x$ & $x$ & $x$ & $x$ & & & & \\
\hline $\begin{array}{l}\text { Pilotrichaceae } \\
\text { Cyclodictyon richardsii Bowers \& Magill }\end{array}$ & & & $x$ & & & & & & & & & \\
\hline $\begin{array}{l}\text { Polytricaceae } \\
\text { Atrichum oerstedianum (Müll.Hal.) Mitt. }\end{array}$ & $x$ & $x$ & $x$ & $x$ & $x$ & $x$ & & & & & & \\
\hline $\begin{array}{l}\text { Pterobryaceae } \\
\text { Hildebrandtiella guyanensis (Mont.) W.R.Buck }\end{array}$ & & & $x$ & $x$ & $x$ & $x$ & $x$ & $x$ & $x$ & & & \\
\hline $\begin{array}{l}\text { Sematophyllaceae } \\
\text { Donnellia commutata (Müll.Hal.) W.R.Buck } \\
\text { Pylaisiadelpha sharpii H.A.Crum } \\
\text { Sematophyllum cuspidiferum Mitt. } \\
\text { Sematophyllum swartzii (Schwägr.) Welch \& H.A.Crum }\end{array}$ & & $X$ & $\begin{array}{l}X \\
X \\
X \\
X\end{array}$ & $\begin{array}{l}X \\
X \\
X\end{array}$ & $\begin{array}{l}X \\
X \\
X\end{array}$ & $\begin{array}{l}X \\
X \\
X\end{array}$ & & $\begin{array}{l}X \\
X \\
X\end{array}$ & & & & \\
\hline
\end{tabular}

Distribución y hábitat. Raíz de Alnus jorullensis Kunth cubiertas con suelo. Especie Americana, en México es conocida de Tamaulipas a Chiapas.

Ejemplares examinados. San Luis Potosí. Mpio. Xilitla, El Retén, 3 de octubre de 2009, P. Herrera 1221.

Campylopus anderssonii (Müll.Hal.) Jaeg., Ber. Thätigk. St. Gallischen Naturwiss. Ges. 1870-71: 436. (Gen. Sp. Musc.
1: 140). 1872. (Dicranaceae). Entre sus características están las hojas que se estrechan gradualmente, no miden más de $10 \mathrm{~mm}$ de largo, con la costa angosta, las células laminares superiores son cortas, rectangulares y en sección transversal muestran hialocistos, además de que las células alares están poco diferenciadas (Frahm, 1991). Los miembros de la familia se caracterizan por ser estrictamente acidófilos, en sustratos con un $\mathrm{pH}<6$ (Frahm, 1991). 
Distribución y hábitat. Sobre Rhus schiedeana Schlecht. y Pinus sp. Por su distribución se le considera Americana. En México, se conocía solamente para Chiapas, Hidalgo y Oaxaca (Sharp et al., 1994; UNIBIO, 2012), por lo que el estado de Querétaro es el límite septentrional de su distribución.

Ejemplares examinados. Querétaro. Mpio. Landa de Matamoros, Los Xililites, San Juan de los Durán, 4 de octubre del 2009, P. Herrera 1307c, 1309c, 1312a, 1488c.

Cyclodictyon richardsii Bowers \& Magill, Bryologist 78: 470.1975. (Pilotrichaceae). Plantas que se caracterizan por ser pequeñas, pues sólo alcanzan hasta $1.5 \mathrm{~cm}$ de largo y 1.5 $\mathrm{mm}$ de ancho con todo y hojas, de un color amarillo-verdoso; sus hojas son ovadas, corto acuminadas, con un borde generalmente de dos células en la base, extendiéndose hasta la parte superior pero sin llegar al ápice; los márgenes foliares son serrulados en la parte distal; en la parte abaxial de la hoja llevan yemas filamentosas (Sharp et al., 1994).

Distribución y hábitat. Sobre Liquidambar styraciflua. Esta es especie endémica de México; la localidad tipo es Xilitla (San Luis Potosí), en BHM. Recientemente, también se le ha encontrado en Veracruz (C. Delgadillo, com. pers.).

Ejemplares examinados. Querétaro. Mpio. Landa de Matamoros, Pozo del Limón, aprox. $3 \mathrm{~km}$ al sur de Neblinas, 21 de octubre de 2011, P. Herrera 1510c.

Donnellia commutata (Müll.Hal.) W.R.Buck, Bryologist 91: 134. 1988. (Sematophyllaceae). Esta especie se puede confundir con Sematophyllum adnatum (Mx.) E.Britton; no obstante, las hojas de $D$. commutata tienen las células alares ligeramente infladas, con las supra-alares rectangulares formando grupos triangulares (Sharp et al., 1994). La distinción inequívoca de la especie es por medio del esporofito, con su seta corta (3-4 mm de largo) y peristoma simple de color blanco en estado seco; dientes ligeramente papilosos distalmente, endostoma ausente o muy corto. En contraste, en S. adnatum el peristoma es doble (Buck, 1988).

Distribución y hábitat. Esta especie se encontró sobre Rhus schiedeana. Con una distribución del Caribe, en México Sharp et al. (1994) la registran para Veracruz. No hay ejemplares de esta especie en México, representadas en MEXU (Delgadillo, 2011).

Ejemplares examinados. Querétaro. Mpio. Landa de Matamoros, Los Xililites, San Juan de los Durán, 4 de octubre de 2009, P. Herrera 1315, $1317 a$.

Entodon hampeanus Müll.Hal., Linnaea 18(6): 705. 1844. (Entodontaceae). Los tallos son fuertemente aplanados, de un color verde brillante, con las hojas agudas y cortamente acuminadas. El esporofito es la característica distintiva de la especie, pues tiene la seta color amarillo, con los dientes del exostoma estriolados externamente en la parte basal y ligeramente papilosos en la distal, mientras que el endostoma es liso o ligeramente papiloso (Sharp et al. 1994; Cárdenas y Delgadillo, 2009).

Distribución y hábitat. Sobre Liquidambar styraciflua. Por su distribución mundial se considera parte del elemento del Caribe (Delgadillo, 2004). En México se distribuye en los estados de Chiapas, Distrito Federal, Oaxaca, Puebla, San Luis Potosí, Tamaulipas y Veracruz (Crum, 1951; Sharp et al., 1994; UNIBIO, 2012).

Ejemplares examinados. Querétaro. Mpio. Landa de Matamoros, Pozo del Limón, aprox. $3 \mathrm{~km}$ al sur de Neblinas, 22 de agosto de 2009, P. Herrera $1184 a ; \pm 3 \mathrm{~km}$ (por aire) al SE de La Cercada, por el camino a La Mesa, 24 de Octubre de 2002, Zamudio y Steinmann 12010 (MEXU).

Entodon jamesonii (Taylor) Mitt., J. Linn. Soc., Bot. 12: 528. 1869. (Entodontaceae). Plantas ligeramente aplanadas, con hojas acuminadas y lanceoladas, serradas, las células alares son cuadradas o cortamente rectangulares y alcanzan la costa en las hojas angostas, pero no en las más anchas. El exostoma es estriolado, casi hasta el ápice; el endostoma liso a ligeramente papiloso (Sharp et al., 1994; Cárdenas y Delgadillo, 2009).

Distribución y hábitat. Sobre Liquidambar styraciflua. Especie Americana; se le ha registrado en 11 estados, incluyendo Hidalgo, Puebla, Tamaulipas y Veracruz (Sharp et al., 1994; UNIBIO, 2012), por lo que era de esperarse su presencia en Querétaro.

Ejemplares examinados. Querétaro. Mpio. Landa de Matamoros, Pozo del Limón, aprox. $3 \mathrm{~km}$ al sur de Neblinas, 21 de octubre de 2011, P. Herrera 1534c.

Entodon serrulatus Mitt., J. Linn. Soc., Bot. 12: 632. 1869. (Entodontaceae). Esta especie se diferencia de la anterior en que las células basales están muy bien desarrolladas y siempre alcanzan la costa, además de que su endostoma es fuertemente papiloso, por lo que es necesaria la presencia del esporofito para su identificación inequívoca (Sharp et al. 1994).

Distribución y hábitat. Sobre Clethra aff. kenoyeri Lundell y Liquidambar styraciflua. Considerada del Caribe, se distribuye en México en los estados de Chiapas, Distrito Federal, Hidalgo, México, Michoacán, Morelos, Oaxaca, Puebla y Veracruz (Sharp et al., 1994; UNIBIO, 2012).

Ejemplares examinados. San Luis Potosí. Mpio. Tamasopo, Copalillos, 18 de marzo de 2010, P. Herrera 1319f, 1341b, 1399e.

Fissidens dubius P.Beauv., Prodr. Aethéogam. 57. 1805. (Fissidentaceae). Es una de las especies más grandes del género (hasta $2.5 \mathrm{~cm}$ de largo), con hojas cuyos márgenes son irregularmente serrados, con las células marginales de un color más claro que las interiores; láminas foliares irregularmente biestratificadas (Sharp et al. 1994).

Distribución y hábitat. Sobre suelo cubriendo rocas. Es una especie de distribución boreal (Delgadillo, 2004); ha sido 
registrada en los estados de Chiapas, Sonora y Veracruz (Pursell, 2007); Hidalgo, Puebla, San Luis Potosi y Tamaulipas (Sharp et al., 1994; UNIBIO, 2012).

Ejemplares examinados. Querétaro. Mpio. Landa de Matamoros, Los Xililites, San Juan de los Durán, 4 de octubre de 2009, P. Herrera 1277.

Fissidens serratus Müll.Hal. var. serratus, Bot. Zeitung (Berlin) 5: 804. 1847. (Fissidentaceae). Entre las características que distinguen a la especie se encuentran su crecimiento palmeado con tallos cortos, hojas lanceoladas y agudas, con márgenes dentados, células unipapilosas y costa percurrente (algunas veces llegando hasta ocho células abajo del ápice); esta variedad no presenta un borde diferenciado en la lámina vaginante (Pursell, 2007).

Distribución y hábitat. Sobre Clethra kenoyeri, Eugenia xalapensis (Kunth) DC., Liquidambar styraciflua y Sambucus sp. Se considera de distribución pantropical (Delgadillo y Cárdenas, 2002). Se le ha registrado en Campeche, Chiapas, San Luis Potosí, Tamaulipas y Veracruz (Sharp et al., 1994; Pursell, 2007; UNIBIO, 2012).

Ejemplares representativos. Querétaro. Mpio. Landa de Matamoros, Pozo del Limón, aprox. $3 \mathrm{~km}$ al sur de Neblinas, 22 de agosto de 2009, P. Herrera 1166b, 1171b; Los Xililites, San Juan de los Durán, 20 de agosto de 2010, P. Herrera 1496c. San Luis Potosí. Mpio. Xilitla, El Retén, 3 de octubre de 2010, P. Herrera 1200c, 1438b; Mpio. Tamasopo, Copalillos, 18 de marzo de 2010, P. Herrera 1322b, 1349 .

Hildebrandtiella guyanensis (Mont.) W.R.Buck, Brittonia 43: 97. 1991. (Pterobryaceae). Sus plantas son estipitadas, en donde las hojas están espiralmente insertadas; las hojas de los tallos son generalmente oblongas y cóncavas, sin costa; las células alares no están muy diferenciadas del resto; usualmente tienen yemas (Sharp et al., 1994).

Distribución y hábitat. Sobre Eugenia xalapensis y Quercus sp. Se le considera de amplia distribución bicéntrica (Delgadillo, 2004). En México se ha reportado para los estados Chiapas, Oaxaca, Sinaloa, Tamaulipas y Veracruz (Sharp et al., 1994; UNIBIO, 2012).

Ejemplares examinados. Querétaro. Mpio. Landa de Matamoros, Los Xililites, San Juan de los Durán, 20 de agosto de 2010, P. Herrera 1466b, 1473, 1475a.

Isopterygium tenerum (Sw.) Mitt., J. Linn. Soc., Bot. 12: 499. 1869. (Hypnaceae). Plantas postradas, complanado-foliadas, verde amarillentas, con pseudoparafilios filamentosos; sus hojas generalmente de menos de $1 \mathrm{~mm}$ de largo, ovado-lanceoladas, acuminadas; las células alares diferenciadas en un grupo pequeño; el esporofito con cápsulas inclinadas u horizontales sobre setas de 5-12 $\mathrm{mm}$ de largo (Cárdenas y Delgadillo, 2009).

Distribución y hábitat. Sobre Clethra sp. y Pinus sp. Especie de amplia distribución. En México es común (Sharp et al.,
1994) y debido a que ya se registraba en localidades cercanas al área de estudio como Xilitla (Sharp 5935; San Luis Potosí; UNIBIO, 2012) era probable encontrarla en Querétaro.

Ejemplares examinados. Querétaro. Mpio. Landa de Matamoros, Pozo del Limón, aprox. $3 \mathrm{~km}$ al sur de Neblinas, 21 de octubre del 2011, P. Herrera 1527a; Los Xililites, San Juan de los Durán, 20 de agosto de 2010, P. Herrera 1489c.

Leucobryum crispum Müll.Hal., Syn. Musc. Frond. 1: 78. 1848. (Leucobryaceae). Se caracteriza porque sus plantas son blanquecino-verdosas, con las hojas crispadas, hasta de $9 \mathrm{~mm}$ de largo; en sección transversal de la parte más ancha de la hoja se encuentran clorocistos con cuatro ángulos y de 3-4 capas de leucocistos (Sharp et al., 1994).

Distribución y hábitat. Sobre suelo y Liquidambar styraciflua, Pinus sp. y Rhus schiedeana. Por su distribución mundial se considera del Caribe y en México sólo se le reportaba en Chiapas (Sharp et al., 1994). Los nuevos registros en Querétaro, San Luis Potosí y Veracruz (C. Delgadillo com. pers.) amplían su distribución.

Ejemplares representativos. Querétaro. Mpio. Landa de Matamoros, Los Xililites, San Juan de los Durán, 4 de octubre de 2009, P. Herrera 1276, 1308a, 1309b, 1490. San Luis Potosí. Mpio. Tamasopo, Copalillos, 18 de marzo de 2010, P. Herrera 1397 c.

Orthostichella pachygastrella (Müll.Hal. ex Ångstr.) B.H.Allen \& Magill, Bryologist 110: 16. 2007. (Neckeraceae). Las plantas de este género son largas, estolonadas, delgadas, los tallos secundarios estipitados e irregularmente ramificados, colgantes; algunas especies como $O$. $p a$ chygastrella tienen hojas deciduas (Allen y Magill, 2007). Este género puede confundirse con Pilotrichella, pero este último es más robusto, sus hojas no tienen costa y las células alares están bien desarrolladas en grupos frecuentemente abultados (Allen y Magill, 2007).

Distribución y hábitat. Sobre Ilex rubra S. Watson y Prunus sp. Por su distribución mundial se le considera Americana. En México se le registra en Chiapas, Veracruz y Tabasco (Allen y Magill, 2007).

Ejemplares examinados representativos. San Luis Potosí. Mpio. Tamasopo, Copalillos, 18 de marzo de 2010, P. Herrera 1360, 1371a.

Pylaisiadelpha sharpii H.A.Crum, Bryologist 89: 27. 1986. (Sematophyllaceae). Esta especie, nombrada en honor de Aaron J. Sharp, se caracteriza por tener hojas menores a 1 $\mathrm{mm}$ de largo, células alares moderadamente infladas, cápsulas inclinadas a péndulas y un endostoma bien desarrollado, con una membrana basal alta y con cilios (Crum, 1986). Algunos autores consideran que las características del peristoma no concuerdan con el concepto genérico (Ando et al., 1989), pero sigue siendo una especie reconocida (Delgadi1lo, 2010). 
Distribución y hábitat. Se le encontró sobre Pinus sp. Esta especie es endémica de México y se le conoce en Chiapas, Michoacán y Puebla (Sharp et al., 1994; Delgadillo y Cárdenas, 2002; UNIBIO, 2012).

Ejemplares examinados. Querétaro. Mpio. Landa de Matamoros, Los Xililites, San Juan de los Durán, 20 de agosto de 2010, P. Herrera $1488 b$.

Schlotheimia jamesonii (Arnott) Brid., Bryol. Univ. 1: 742. 1826. (Orthotrichaceae). Esta especie se parece a $S$. rugifolia (Hook.) Schwägr., pero generalmente sus hojas no son rugosas y son oblongo-lanceoladas, acuminadas o con un apículo ancho; las hojas periqueciales están diferenciadas, hasta $3.4 \mathrm{~mm}$ de largo y altamente envainantes (Sharp et al., 1994).

Distribución y hábitat. Sobre Crataegus mexicana DC., Liquidambar styraciflua, Quercus sp. y Rhus schiedeana. Por su distribución mundial se considera del Caribe, y en México se le registra para los estados de Oaxaca, San Luis Potosí, Tamaulipas y Veracruz (Sharp et al., 1994; UNIBIO, 2012). Ejemplares representativos examinados. Querétaro. Mpio. Landa de Matamoros, Los Xililites, San Juan de los Durán, 4 de octubre de 2010, P. Herrera 1269, 1278b, 1302a.

Sematophyllum cuspidiferum Mitt., J. Linn. Soc., Bot. 12: 480. 1869. (Sematophyllaceae). Se caracteriza por su hábito postrado y ser de tamaño grande, en comparación con las otras especies del género, de un color amarillo a dorado verdoso; las hojas son cóncavas, oblongo-ovadas y subuladas con el acumen plano (Sharp et al., 1994).

Distribución y hábitat. Sobre Clethra pringlei S.Watson, Liquidambar styraciflua, Salvia sp. y Zanthoxylum sp. Por su distribución mundial se considera del Caribe (Delgadi1lo, 2004), y en México se ha registrado en varios estados que forman parten de la Sierra Madre Oriental (p. ej. Hidalgo, Tamaulipas y Veracruz) (Sharp et al., 1994; UNIBIO, 2012).

Ejemplares examinados. Querétaro. Mpio. Landa de Matamoros, Los Xililites, San Juan de los Durán, 20 de agosto de 2010, P. Herrera 1496f. San Luis Potosí. Mpio. Xilitla, El Retén, 20 de marzo de 2010, P. Herrera 1452, 1456a, 1462a; $3 \mathrm{~km}$ al W de Ahuacatlán, 22 de Agosto de 1978, Cárdenas 147 (MEXU); Mpio. Tamasopo, Copalillos, 18 de marzo de 2010, 18 de marzo del 2010, P. Herrera 1419c.

Sematophyllum swartzii (Schwägr.) Welch \& H.A.Crum, Bryologist 62: 176. 1959. (Sematophyllaceae). La principal característica que distingue a esta especie de las otras del género son sus hojas estrechamente acuminadas y falcadassecundas (Cárdenas y Delgadillo, 2009).

Distribución y hábitat. Sobre Clethra pringlei y Liquidambar styraciflua. Su distribución mundial la ubica dentro del elemento del Caribe (Delgadillo, 2004). Se ha registrado en varios estados (p. ej. Guerrero, Hidalgo, Michoacán, Sina- loa; Sharp et al., 1994) y tipos de bosque, incluyendo los BHM, como en el Rancho El Cielo (Tamaulipas) (UNIBIO, 2012).

Ejemplares examinados. Querétaro. Mpio. Landa de Matamoros, Los Xililites, San Juan de los Durán, 20 de agosto de 2010, P. Herrera 1492a; $\pm 3 \mathrm{~km}$ (por aire) al SE de La Cercada, por el camino a La Mesa, 24 de Octubre de 2002, Zamudio y Steinmann 12100 (MEXU). San Luis Potosí. Mpio. Xilitla, El Retén, 20 de marzo de 2010, P. Herrera 1426a, 1427a.

Sphaerotheciella pachycarpa (Schimp. ex Besch.) Manuel, J. Hattori Bot. Lab. 49: 136. 1981. (Cryphaceae). El aspecto de esta planta es similar al de Cryphaea, pero en el esporofito los dientes del peristoma son lisos o en la base, lisos y ligeramente papilosos distalmente; las esporas germinan precozmente en la urna (Sharp et al., 1994). Las hojas de esta especie son generalmente ovadas a elípticas y, agudas a acuminadas y enteras o ligeramente serruladas en el ápice (Sharp et al., 1994; Rao, 2000).

Distribución y hábitat. Sobre Zanthoxylum sp. De distribución Mesoamericana, en México se le ha encontrado en Chiapas, Hidalgo, México, Oaxaca, Puebla y Vercruz (Sharp et al., 1994; UNIBIO, 2012).

Ejemplares examinados. San Luis Potosí. Mpio. Xilitla, El Retén, 20 de marzo de 2010, P. Herrera 1457a.

Squamidium leucotrichum (Taylor) Broth., Nat. Pflanzenfam. I: 809. 1906. (Meteoriaceae). Ayuda a identificar a la especie el hábito colgante, con hojas oblongas a abruptamente subuladas terminando en un pelo, con costa, células lisas, con un grupo de células alares pequeño pero evidente (Sharp et al., 1994).

Distribución y hábitat. Sólo se encontró una muestra exigua sobre Quercus germana Schltdl. \& Cham. Por su distribución mundial se considera del Caribe y se le ha registrado en los estados de Chiapas, Michoacán, Oaxaca, Puebla y Veracruz (Sharp et al., 1994; UNIBIO, 2012).

Ejemplares examinados. San Luis Potosí. Mpio. Tamasopo, Copalillos, 18 de marzo de 2010, P. Herrera 1396c.

Syrrhopodon incompletus Schwägr. var. incompletus, Sp. Musc. Frond., Suppl. 2: 119. 1824. (Calymperaceae). En ausencia de esporofitos y de teniola, algunas especies se pueden confundir con Calymperes. En esta variedad la lámina tiene el borde engrosado y dentado; las cancelinas son persistentes y la base foliar hialina con hombros poco prominentes; yemas en los ápices de las hojas (Allen, 1994; Sharp et al., 1994).

Distribución y hábitat. Se le encontró sobre Clethra pringlei. Esta variedad de amplia distribución bicéntrica (América-África) (Delgadillo, 2004) se ha registrado tanto en el sur del país (p. ej. Yucatán) como en el norte (Tamaulipas) (Sharp et al., 1994; UNIBIO, 2012). 
Ejemplares examinados. Querétaro. Mpio. Landa de Matamoros, Pozo del Limón, aprox. $3 \mathrm{~km}$ al sur de Neblinas, 21 de octubre de 2011, P. Herrera 1526b, $1539 b$.

Syrrhopodon parasiticus (Brid.) Besch., Ann. Sci. Nat., Bot., sér. 8, 1: 298. 1895. (Calymperaceae). Las plantas de esta especie tienen la teniola incompleta y las hojas, en estado seco, son ligeramente torcidas; las características distintivas son las hojas liguladas-lanceoladas, con las células dorsalmente unipapilosas y las yemas ubicadas en la superficie adaxial a lo largo de la costa (Allen, 1994; Sharp et al., 1994).

Distribución y hábitat. Sobre Clethra pringlei, Crataegus mexicana y Rhus schiedeana. Esta especie de distribución pantropical (Delgadillo, 2004) se ha encontrado en los estados de Campeche, Chiapas, Jalisco, Nayarit, Puebla, Quintana Roo, Veracruz y Yucatán (Sharp et al., 1994; UNIBIO, 2012).

Ejemplares examinados. Querétaro. Mpio. Landa de Matamoros, Los Xililites, San Juan de los Durán, 4 de octubre de 2009, P. Herrera 1293d, 1316c, 1318c, 1494.

Syrrhopodon prolifer Schwägr. var. prolifer, Sp. Musc. Frond., Suppl. 2: 99. pl. 180. 1827. (Calymperaceae). A diferencia de las especies anteriores, en ésta las hojas están más rectas cuando secas, de un color verde; presenta un borde de células hialinas alargadas en toda la hoja, con los hombros de las hojas ligeramente marcados, pero irregular y ligeramente dentados, con la lámina distal más larga que la base; las células son pluripapilosas en ambas superficies (Allen, 1994; Sharp et al., 1994). Aunque generalmente no presentan yemas, un ejemplar sí las mostró.

Distribución y hábitat. Sobre Liquidambar styraciflua y Sambucus sp. También es de distribución pantropical, en México se le ha registrado en Chiapas, Hidalgo, Nayarit, Oaxaca, Puebla, San Luis Potosí, Tamaulipas y Veracruz (Sharp et al., 1994; UNIBIO, 2012).

Ejemplares examinados. Querétaro. Mpio. Landa de Matamoros, Pozo del Limón, aprox. $3 \mathrm{~km}$ al sur de Neblinas, 22 de agosto de 2009, P. Herrera 1165, $1532 a$.

\section{Discusión}

Con los nuevos registros, la flora de musgos del estado de Querétaro comprende ahora 225 especies y 221 para San Luis Potosí. Sin embargo, el número de especies para esta última entidad se debe tomar con prudencia, porque aunque se revisó la información taxonómica pertinente de las especies registradas en este trabajo, es necesaria la actualización nomenclatural de las especies de musgos mencionadas por Sharp et al. (1994). No obstante, tomando en cuenta esta riqueza de musgos y considerando las 983 especies reconocidas para México (Delgadillo, 2011), en estas entidades está representada el $22.88 \%$ (Querétaro) y el $22.48 \%$ (San
Luis Potosí) de la flora de musgos del país. Esta diversidad, aunque no comparable con la de los estados de Chiapas, Oaxaca y Veracruz (Delgadillo, 2011), es significativa por los taxones que contiene.

De los nuevos registros, dos especies son endémicas de México, como Cyclodictyon richardsii que sólo se le localiza, hasta ahora, en el centro de la Sierra Madre Oriental. En cambio, Pylaisiadelpha sharpii, aunque se le conoce en otros estados del país, se le considera un taxón inconspicuo por los pocos registros existentes en MEXU.

Donnellia commutata, una especie incluida en la flora de musgos de México pero sin ejemplares de referencia en MEXU, se encontró en el área de estudio. Esta especie se colectó en el tronco de Rhus schiedeana, a una altura mayor a los $1.3 \mathrm{~m}$ del suelo, lo cual coincide con lo descrito por Buck (1988), quien menciona que es frecuente a esta altura y hasta los $2.5 \mathrm{~m}$ del suelo. Por lo anterior, y aunado a las características taxonómicas, las ecológicas también son necesarias de tomar en cuenta para distinguirla de Sematophyllum adnatum, que se puede encontrar en cualquier parte del árbol.

Los otros nuevos registros son comparativamente mejor conocidos en otras áreas del país, pero su presencia en Querétaro y San Luis Potosí aumenta su área de distribución y sugiere que dichas entidades requieren una mejor caracterización de la flora de musgos. La protección de un área por su riqueza de plantas vasculares y vertebrados no siempre garantiza la conservación de la diversidad de briofitas (Hallingbäck y Hodgetts, 2000; Chiarucci et al., 2007). Por el momento, la presencia de las especies señaladas indica la importancia de los BHM de la región terrestre prioritaria Sierra Gorda-Río Moctezuma.

Aunque son pocas las especies tratadas en este trabajo, no sorprende que su área de distribución sea más bien tropical; la presencia de especies de distribución boreal muestra que, entre los musgos, la mezcla de especies tropicales y templadas en el bosque húmedo de montaña también es frecuente, como lo señala Delgadillo (1979). La continuación de los estudios sobre la flora regional de musgos y su distribución son aún necesarios para documentar su presencia y su significado ante la pérdida de hábitats en nuestro país.

\section{Agradecimientos}

A Claudio Delgadillo, quien revisó el manuscrito y proporcionó valiosos comentarios, además de resolver dudas taxonómicas. Ángeles Cárdenas apoyó con la identificación de algunos ejemplares. Hugo A. Castillo apoyó en el trabajo de campo y en la determinación de las plantas leñosas. Enrique Ortíz Bermudes elaboró el mapa. A Deneb García y a un revisor anónimo, por sus correcciones, comentarios y sugerencias. La primera autora agradece el apoyo de la Universidad Autónoma de Querétaro y la beca del CONACYT (48348). 


\section{Literatura citada}

Arriaga L., Espinoza J.M., Aguilar C., Martínez E., Gómez L. y Loa L. Coords. 2000. Regiones terrestres prioritarias de México. Comisión Nacional para el conocimiento y Uso de la Biodiversidad. <http://www.conabio.gob.mx/conocimiento/regionalizacion/doctos/Tlistado.html> (consultado en mayo 2012).

Allen B. 1994. Moss Flora of Central America. Part 1. Sphagnaceae-Calymperaceae. Monographs in Systematic Botany from the Missouri Botanical Garden 49:1-242.

Allen B. y Magill R.E. 2007. A revision of Orthstichella (Neckeraceae). The Bryologist 110:1-45.

Ando H. y Higuchi M. 1983. Homomallium sharpii, a new moss species from Mexico. The Bryologist 86:374-377.

Ando H., Seki T. y Schofield W.B. 1989. Generic distinctness of Brotherella from Pylaisiadelpha (Musci). The Bryologist 92:209-215.

Buck W.R. 1988. Donnellia (Sematophyllaceae) resurrected and refound in Florida after 110 years. The Bryologist 91:134-135.

Buck W.R. 1998. Pleurocarpous Mosses of the West Indies. Memoirs of the New York Botanical Garden 82:1-400.

Cárdenas S.A. 1999. Los musgos pleurocárpicos del Valle de México, México. Tropical Bryology 16:109-116.

Cárdenas S.A. y Delgadillo M. C. 2009. Musgos del Valle de México. Cuadernos del Instituto de Biología Vol. 40, Universidad Nacional Autónoma de México, México, D.F.

Chiarucci A., D'auria F. y Bonini I. 2007. Is vascular plant species diversity a predictor of bryophyte species diversity in Mediterranean forest? Biodiversity and Conservation 16:525-545.

CrumH.A. 1951. Index of Appalachian-Ozarkianelement in the mosses flora of Mexico with a check-list of all know Mexican mosses. Tesis doctoral. University of Michigan, Ann Arbor. 504 pp.

Crum H. 1986. Taxonomic and nomenclatural addenda to the Mexican moss flora. The Bryologist 89:23-27.

Delgadillo M.C. 1979. Mosses and phytogeography of the Liquidambar forest of Mexico. The Bryologist 82:432-449.

Delgadillo M.C. 1984. Mosses of the Yucatan Peninsula, Mexico. III. Phytogeography. The Bryologist 87:12-16.

Delgadillo M.C. 1987. Moss distribution and the phytogeographical significance of the Neovolcanic Belt of Mexico. Journal of Biogeography 14:69-78.

Delgadillo M.C. 1998. Diversidad de la brioflora mexicana. En: Ramamoorthy T.P., Bye R., Lot A. y Fa J. Eds. Diversidad Biológica de México, Orígenes y Distribución, pp. 355-368, Instituto de Biología, Universidad Nacional Autónoma de México, México, D.F.

Delgadillo M.C. 2004. Musgos. En: Luna I., Morrone J.J. y Espinosa D. Eds. Biodiversidad de la Sierra Madre Oriental, pp. 127-135, Las Prensas de Ciencias, Facultad de Ciencias, Universidad Nacional Autónoma de México, México, D.F.
Delgadillo M.C. 2010. LATMOSS 2010. <http://www.ibiologia. unam.mx/briologia/www/index/latmoss.html > (consultado en agosto 2012).

Delgadillo M.C. 2011. Los musgos, Veracruz y el corredor florístico del Golfo. En: Cruz A.A. Ed. La Biodiversidad en Veracruz. Estudio de Estado. Vol. II. pp. 89-96, Comisión Nacional para el Conocimiento y Uso de la Biodiversidad, Gobierno del Estado de Veracruz, Universidad Veracruzana, Instituto de Ecología, A.C., México, D.F.

Delgadillo M.C. y Cárdenas S.A. 1996. A preliminary checklist of the mosses of Guanajuato, Mexico. Flora del Bajío y Regiones Adyacentes. Fascículo Complementario XI. Comisión Nacional para el Conocimiento y Uso de la Biodiversidad, Instituto de Ecología, A.C., Centro Regional del Bajío, Pátzcuaro.

Delgadillo M.C. y Cárdenas S.A. 2002. The Lacandon forest (Chiapas, México): a benchmark area for tropical mosses. The Bryologist 105:327-333.

Frahm J.P. 1991. Dicranaceae: Campylopodioideae, Paraleucobryoideae. Flora Neotropica 54:1-238.

GBIF. Global Biodiversity Information Facility. 2013. < data.gbif. org/welcome.htm > (consultado en febrero 2013).

Hallingbäck T. y Hodgetts N. 2000. Key habitats and their specific threats and recommendations. En: Hallingbäck T. y Hodgetts N. Comps. Mosses, Liverworts and Hornworts. Status Survey and Conservation Action Plan for Bryophytes, pp 14-21, IUCN, SSC Bryophyte Specialist Group, Gland and Cambridge.

Juárez G.G. 1983. Los musgos de Coatepec, Veracruz, México. Biótica 8:49-58.

Herrera-Paniagua P., Delgadillo M.C., Villarseñor R.J.L. y LunaVega I. 2008. Floristics and biogeography of the mosses of the state of Querétaro, Mexico. The Bryologist 111:41-56.

Pursell R.A. 2007. Fissidentaceae. Flora Neotropica 101:1-278.

Rao P. 2000. Sphaerotheciella koponenii (Bryopsida, Cryphaceae), a new species from China. The Bryologist 103:739-741.

Sharp A.J., Crum H. y Eckel P.M. Eds. 1994. The Moss Flora of Mexico. Memoirs of the New York Botanical Garden 69:11113.

Thornburgh K.R. y Sharp A.J. 1975. A preliminary list of mosses collected on tree branches in Mexico. Boletín de la Sociedad Botánica de México 35:51-58.

UNIBIO. Unidad de Informática para la Biodiversidad. 2012. Colecciones Biológicas Nacionales. Instituto de Biología, Universidad Nacional Autónoma de México. <http://www.unibio. unam.mx> (consultada en mayo 2012).

Villaseñor J.L. 2010. El Bosque Húmedo de Montaña en México y sus Plantas Vasculares: Catálogo Florístico-Taxonómico. Comisión Nacional para el Conocimiento y Uso de la Biodiversidad, Universidad Nacional Autónoma de México, México, D.F. 